Vol. 6, $n^{\circ} 1 \mid 2002$

Varia

\title{
La Justice en France, 1789-1939. Un modèle à l'épreuve
}

Frédéric Chauvaud

\section{(2) OpenEdition \\ Journals}

Édition électronique

URL : https://journals.openedition.org/chs/240

DOI : $10.4000 /$ chs. 240

ISSN : 1663-4837

Éditeur

Librairie Droz

Édition imprimée

Date de publication : 1 juillet 2002

ISBN : 2-600-00803-9

ISSN : 1422-0857

\section{Référence électronique}

Frédéric Chauvaud, "La Justice en France, 1789-1939. Un modèle à l'épreuve », Crime, Histoire \&

Sociétés / Crime, History \& Societies [En ligne], Vol. 6, n¹ | 2002, mis en ligne le 03 février 2009, consulté le 22 mars 2022. URL : http://journals.openedition.org/chs/240 ; DOI : https://doi.org/ $10.4000 /$ chs. 240

Ce document a été généré automatiquement le 22 mars 2022

(c) Droz 


\title{
La Justice en France, 1789-1939. Un modèle à l'épreuve
}

\author{
Frédéric Chauvaud
}

1 La France incarne l'un des modèles européens d'État ancien et centralisé, elle représente aussi le creuset ${ }^{2}$ de la "révolution de la justice $»^{3}$ qui se traduit par la mise en place soudaine d'un nouveau "système des tribunaux $»^{4}$. Pour illustrer la modernisation de la justice, Pierre Bouchardon usa en 1926 d'une singulière métaphore. La rationalisation de la justice et la simplification de la législation se sont concrétisées par un processus de miniaturisation. Jadis, il fallait des bibliothèques "hautes de dix coudées» pour contenir l'ensemble des textes juridiques avec "leurs scolies et leurs dérivés "; aujourd'hui tous les codes sont disponibles sous un format minuscule, ils sont devenus des «livres de gousset ", que l'on peut emmener avec soi, partout où nos pas nous dirigent ${ }^{5}$. Si personne ne songe à nier l'évidence et l'importance d'une transformation d'ampleur et d'une rationalisation effective, il n'en reste pas moins que l'étatisation de la justice, voire sa "standardisation", n'ont pas été totalement accomplies en 1790 ou en 1810 et qu'elles n'ont pas davantage recueilli l'unanimité. Sans doute alors, convient-il de déjouer les ruses de l'imaginaire politique et juridique. Les «masses de granit» napoléoniennes ont été décrétées sans pour autant être acceptées ou appliquées sur l'ensemble du territoire.

2 Les résistances à la centralisation de la justice, les tactiques de contournement ou d'évitement à l'égard d'une institution judiciaire étatisée, les manières d'apprivoiser le "système des tribunaux» ou de s'y opposer ont été nombreuses et ont emprunté de multiples chemins, mais elles n'ont donné lieu, jusqu'à présent, qu'à une poignée de travaux parcellaires ${ }^{6}$. Aussi, puisque seuls quelques " prélèvements d'échantillons $»^{7}$ sont disponibles, l'histoire de la contestation d'un "modèle de justice", entre la révolte ouverte, la désobéissance feutrée, sans oublier les critiques vipérines, ne peut être ici qu'esquissée.

3 Sans doute convient-il alors de partir d'abord du localqui est assurément l'une des entrées les plus pertinentes pour comprendre comment fonctionne une société nationale, en l'occurrence, la nation France, qui ne peut être dissociée d'un modèle 
étatique. Une telle démarche oblige en tout cas à changer d'échelle et à ne pas s'attacher seulement à une histoire institutionnelle vue d'en haut. En effet, dans cette dernière perspective, la marche de la justice s'apparente à un processus de rationalisation, homogène et global, qui s'impose sur l'ensemble du territoire national. Depuis le XIII ${ }^{\mathrm{e}}$ siècle, l'État aurait monopolisé à son profit la violence, aurait assuré la paix à l'intérieur et exporté la guerre aux confins de ses frontières, aurait élargi son domaine d'intervention, faisant reculer sans cesse les limites entre le privé et le public, mettant en place, à son profit, des mécanismes d'obéissance ${ }^{8}$. Dans cette optique, l'État et l'institution judiciaire sont en effet perçus et décrits à partir de la fin de l'Ancien Régime comme, d'une part, des entités fonctionnelles et nécessaires et, d'autre part, comme des instances plus complexes, puisqu'elles se spécialisent, tout en étant davantage transparentes ${ }^{9}$. De la sorte, tous les «ingrédients » seraient en place pour que l'État autoritaire napoléonien puisse s'effacer, à la suite d'évolutions lentes ou tumultueuses, devant un État républicain, réussissant la synthèse du libéralisme et de la démocratie ${ }^{10}$. Pourtant, le modèle français d'une justice centralisée et étatisée est en réalité contesté avec plus ou moins d'ampleur dès 1790 ou dès l'an VIII, même si par ailleurs la justice a d'emblée toutes les allures d'une «forteresse » immobile que rien ne semble devoir ébranler.

4 Reste que le modèle judiciaire français a fonctionné et a duré. Il a incarné notamment la « révolution pénale » contemporaine ${ }^{11}$. Il a même été « exporté $~^{12}$. Pour autant, il n'a jamais fait l'unanimité et il a semblé pendant très longtemps incompatible avec l'esprit républicain ou avec les modes de régulation des communautés villageoises. Trois approches sont ici privilégiées: la géographie, le local et les justiciables. Elle ne prétendent pas épuiser le sujet, mais sans doute permettent-elles de mieux comprendre les réticences et les résistances, les rythmes différés et décalés de mise en place du modèle de justice étatisée. Sans doute encore permettent-elles de saisir la défiance grandissante à l'égard du fonctionnement des juridictions et de l'État ${ }^{13}$, amenant une partie de l'opinion publique à se demander s'il existe même un modèle républicain de la justice.

\section{La géographie contre l'uniformisation judiciaire}

5 La géographie historique a subi pendant longtemps une éclipse due à la surdétermination descriptive du territoire et au discrédit d'une histoire politique ou d'une géographie électorale arrimées au sous-sol, épousant le relief et expliquant les manières d'agir des acteurs locaux par les spécificités topographiques. Depuis, dans des champs très dissemblables, cette discipline a connu un important renouveau ${ }^{14}$. Néanmoins, la géographie historique judiciaire n'a guère suscité de travaux, malgré quelques analyses proposées pendant la période de l'entre-deux-guerres ${ }^{15}$ et en dehors des recherches récentes sur la carte judiciaire ${ }^{16}$. Et pourtant, elle seule, dans certaines circonstances, en dévoilant les «structures invisibles " permet de comprendre de quelle manière l'État échoue à imposer sa marque unificatrice, à niveler les particularismes et à modifier les comportements des justiciables.

6 En 1836, l'Essai sur la statistique de la population française retentit avec fracas. Il vient à son heure et davantage que la poignée de travaux qui l'ont précédé et qui bien souvent ont été accueillis avec indifférence, il est le prélude à une prise de conscience collective. La France ne serait pas une mais multiple. L'épisode révolutionnaire n'aurait pas 
permis la constitution d'un cadre commun et monochrome. En effet, les chiffres du recrutement, les dossiers fiscaux, l'esprit de chicane, le nombre de suicides, les crimes contre les personnes et les biens... nombre d'informations encore sont débusquées, recueillies et mises en tableau. Les données permettent de classer chaque département selon son plus ou moins grand degré de moralité. S'esquisse ainsi une représentation spatiale des infractions, des contentieux et dans une certaine mesure de l'activité judiciaire. Même si les « régions ", les "provinces », les " pays » sont relégués, pour un temps, au magasin des curiosités du passé, le découpage départemental utilisé pour l'établissement de statistiques ne parvient pas à gommer les singularités. Le "département ${ }^{17}$, symbole de la modernité administrative, incarne la seule circonscription que l'État reconnaît, et pourtant il ne parvient pas à masquer l'extrême bigarrure du territoire sur lequel il a étendu son emprise.

Dans cette perspective, et en forçant un peu le trait, l'histoire de l'unification du territoire judiciaire peut être lue tantôt comme un échec partiel tantôt comme une réussite tardive et relative. Tout se passe en effet comme si le "résidu juridique " d'époques révolues résistait à toute entreprise de réforme et d'homogénisation.

Deux éclairages, parmi d'autres, permettent d'en rendre compte. Au civil, l'œuvre de codification que l'on voulait commune à tout le royaume puis à la totalité de l'espace français bute sur les legs du passé. Au pénal, les pratiques quotidiennes des plus hautes juridictions répressives s'écartent des recommandations de la Chancellerie. Mises en rapport avec la cartographie judiciaire, elles hantèrent l'imaginaire des spécialistes de la morale et du crime ${ }^{18}$. Elles soulignent d'emblée que la France est composite et qu'elle ressemble davantage à une marquetterie de communes qu'à un espace unitaire. Ainsi, entre les directives de la justice centrale et les pratiques, existe un espace qui, au gré des circonstances, se dilate. Son existence seule est le témoin muet des insuccès de la centralisation judiciaire.

9 Dans ce cadre, convier l'enquête de Bernard Schnapper qui a voulu mesurer non pas l'activité des juges mais l'esprit processif, c'est-à-dire la volonté d'intenter une action ${ }^{19}$, s'impose. Pour cela il a fallu calculer un taux de litigiosité primaire (justices de paix) et un taux de litigiosité secondaire (tribunaux d'arrondissement). Dans le même temps, la centralisation de la justice revient à faciliter l'accès à la justice, comme en témoigne notamment la loi de 1851 sur l'assistance judiciaire ${ }^{20}$. En effet, en moyenne annuelle, le nombre de demandes d'assistance admises par les bureaux d'arrondissement double tous les dix ans jusqu'à l'orée du XXe siècle. D'une certaine façon, l'État central a donc favorisé les plus démunis par l'entremise d'instances locales sans pour autant que l'on assiste à un gonflement des contentieux.

10 Si l'on fonde dans une même catégorie les différentes affaires devant les justices de paix et les tribunaux civils, on obtient un taux global de litigiosité. La tendance d'ensemble n'est donc pas, contrairement aux prévisions, à la hausse mais au reflux, modeste certes, mais incontestable. La seule explication possible réside dans la diversité régionale et nécessite d'avoir recours à la géographie. De la sorte, de grandes différences entre régions sont aisément repérables. Les chiffres globaux à l'échelon national ${ }^{21}$ masquent la diversité des modèles locaux. Certains espaces connaissent une montée, presque ininterrompue de la litigiosité; d'autres, en revanche, s'affaissent et se replient sur eux-mêmes. Toutefois, si on ne prend en compte que la litigiosité secondaire, la tendance au cours du siècle va vers l'atténuation des différences régionales, Mais on ne peut proposer de généralisation hâtive, car l'étude de la 
litigiosité primaire offre un tout autre visage. Les zones de forte litigiosité ont tendance à se transformer. À tel point qu'apparaissent en 1900-1904 de nouveaux espaces de litigiosité "dans des régions autrefois plutôt placides». Au final pourtant, malgré les évolutions, ce sont les permanences qui l'emportent. En effet, "au point de vue procédural comme à bien d'autres, il y a une France du midi et une France du nord, une France des montagnes et une France des plaines ${ }^{22}$. De la sorte, la démonstration constitue une nouvelle illustration du fait que "l'espace national est conçu sur le mode de couples opposés ${ }^{23}$. Reste à l'expliquer. La géographie électorale, la sociologie religieuse, l'urbanisation et de nombreuses autres variables ne permettent pas de trouver une logique. La seule explication qui résiste à l'analyse réside dans le fait que c'est dans les départements dotés des plus forts taux de litigiosité que se trouve le plus grand nombre des anciens pays de droit écrit; inversement, parmi les départements les moins processifs se rencontre le plus grand nombre de pays de droit oral. D'où l'échec apparent, après un siècle, d'uniformisation des pratiques qui résistent à tout projet national. Or il faut bien convenir que dès 1822, dans un autre registre, Basterrèche mettait en garde contre l'existence de la « ligne Magiolo », faille qui divisant la France en deux de Saint-Malo à Genève, présentait potentiellement une sourde menace contre l'idée de nation. Si le Midi, selon l'argumentation déployée à cette occasion et revisitée par Francis Démier, ne fait pas tout à fait partie de la France -il ne serait «qu'associé »- il aurait le droit, s'il ne reçoit pas ce à quoi il aspire, "de prendre ses distances avec une "Nation» qui aurait perdu de facto son caractère consensuel $»^{24}$. Certes les deux lignes de la litigiosité et de la coupure nord/sud se chevauchent sans être identiques, leur tracé se déplace selon les moments et les éléments comparés, il n'en reste pas moins qu'elles posent la question de la cohésion spatiale : n'a-t-on pas, sur le plan judiciaire comme sur d'autres, davantage rassemblé qu'unifié ?

11 Plus récemment, Gilles Rouet, a repris le dossier de la répartition spatiale selon une analyse multivariée ${ }^{25}$. Sa démarche vise à délaisser la logique institutionnelle, pour partir de la demande sociale de la justice. Toujours est-il que le constat est identique. Dans la première moitié du XIX siècle, en fonction des critères retenus, deux France coexistent : une France du Sud dont la processivité se détache nettement et une France $\mathrm{du}$ Nord en retrait. Pour 1880, la réalisation d'une nouvelle coupe semble pertinente, mais la cartographie judiciaire obtenue ne présente guère de différences avec celle de 1840. Le même phénomène se reproduit en 1914. En revanche, en 1930, la situation spatiale est tout autre. Une véritable métamorphose s'est accomplie. La rupture dans les manières de se comporter se situe donc non pas à la veille du premier conflit mondial mais au cours de l'entre-deux-guerres. Il s'agit alors «d'une transformation des modes de recours aux institutions judiciaires, qui accompagne l'évolution des motifs de litiges. La France ne devient pas moins procédurière, mais les justiciables ne trouvent plus (...) de réponse adaptée à leur problème $»^{26}$. D'où un paradoxe, en même temps que se dessine une plus grande présence de l'État, «les conciliations privées" glissent vers un arrière-plan historique.

12 Du côté de la justice pénale, les quelques études sur les décisions des jury ${ }^{27}$ renseignent sur le hiatus entre les consignes du ministère de la Justice et les usages que font les jurés de leurs prérogatives. Parce qu'il s'agit des infractions les plus graves et les plus spectaculaires, les crimes envahissent l'imaginaire social ${ }^{28}$ et cristallisent la "question sécuritaire ${ }^{29}$. À la fin du XIX siècle l'État est évalué à l'aune de son efficacité répressive $\mathrm{e}^{30}$. Le ministère public semble plus particulièrement préoccupé du problème de l'homogénéisation des décisions de justice. La Gazette des tribunaux, miroir à 
multiples facettes de la société judiciaire, contribue à relativiser la règle nationale. À plusieurs reprises, la rédaction du périodique montre que les principes édictés par le législateur ne sont pas appliqués. Lorsque les jugements sont cassés, renvoyés devant une autre juridiction, le quotidien judiciaire met en lumière pour son lectorat la part de subjectivité des cours d'assises: "quel changement de position à quelques mois de distance, quelle différence dans les deux décisions! $»^{31}$. L'opinion des jurés comme les décisions de justice sont présentées comme entachées de subjectivité. La justice rendue de manière identique sur l'ensemble du territoire relèverait ainsi du mythe. Le jury de jugement ${ }^{32}$, héritage du système des deux jurys de la Révolution française, n'est pas assujetti aux magistrats professionnels qui eux-mêmes recevraient leurs instructions directement de la Chancellerie. À partir de 1824 puis surtout de la réforme de 1832, complétée par celle de 1863, à son tour étoffée par le décret du 27 novembre 1870, les circonstances atténuantes et la nouvelle économie des incriminations ${ }^{33}$ permettent de s'intéresser à la personnalité des accusés et donnent un autre statut à la notion de culpabilité. Ce sont les jurés qu, localement, dans l'enceinte de chaque juridiction répressive, décident du degré de culpabilité de celui qui se trouve dans le box des accusés. Dans l'enceinte de la cour d'assises de Versailles, on se lamente : "cette absence de fermeté se démontre encore par le soin pris dès les premiers jours, par le jury, d'écarter les circonstances aggravantes les mieux établies (...) le jury, se rendant en quelque sorte le maître du procès, faisait une composition avec la justice... $»^{34}$. Nombre de magistrats semblent frappés de stupeur. Quelques-uns dénoncent avec effroi l'extension de la catégorie des "criminels à responsabilité atténuée " qui serait un véritable "monstre "...5 comme l'affirme J. Maxwell, à la fois docteur en médecine, procureur général à Bordeaux et chantre de la théorie de la défense sociale. Parce que le jury échappe à la tutelle de la magistrature, il se montre fantasque, dénoncent des voix de plus en plus nombreuses. Si pour Tocqueville, le jury est "un des modes de la souveraineté $d u$ peuple ${ }^{36}$, il est néanmoins très sévèrement brocardé à la charnière du $\mathrm{XIX}^{\mathrm{e}}$ et du XX $\mathrm{XX}^{\mathrm{e}}$ siècle. De toutes parts de vives critiques s'élèvent. Une majorité n'hésite pas à prophétiser sa disparition, ne vient-il pas d'être supprimé en Espagne ? Pour Gabriel Tarde, on ne peut pourtant ni réformer le jury ni le supprimer même si «lignorance, la peur, la naïveté, la versalité, l'inconséquence, la partialité tour à tour servile ou frondeuse, des jurés sont prouvées surabondamment $»^{37}$. Ceux de Bretagne se montrent exagérément "féroces » à l'égard de l'infanticide ${ }^{38}$; ceux de la Loire peuvent acquitter, "malgré l'évidence des preuves », une fille qui a assassiné sa mère pour la voler; ceux du Poitou peuvent sanctionner des aliénés; ceux du Maine-et-Loire et de la Mayenne font preuve, plus particulièrement à la fin du XIX $\mathrm{X}^{\mathrm{e}}$ siècle, d'une plus grande sévérité que la moyenne nationale lorsqu'il s'agit de condamner les auteurs de viols ou d'attentats à la pudeur ${ }^{39}$. Si les mêmes crimes, indépendamment des taux de criminalitét ${ }^{40}$, commis dans des contextes similaires ne sont pas sanctionnés de façon identique, n'est-ce pas l'indice que la justice, malgré son apparente centralisation, n'est pas rendue de manière uniforme et qu'elle est capricieuse $^{41}$ ? Quelques rares contemporains, prenant le contre-pied de la centralisation jacobine, s'empareront de la question de la "déconcentration » qui suggère que certains problèmes doivent être traités par le local. Peut-on faire fonctionner de manière identique des juridictions dont le ressort géographique, la population, l'activité économique, les traditions, le nombre d'affaires et leur nature présentent de grandes différences? Pour d'autres au contraire, il convient de parachever l'étatisation et la modernisation de la justice ${ }^{42}$, ne convient-il pas de 
s'approprier les termes du débat de 1908 relatif à la création d'« une police d'État complètement centralisée et indépendante des influences locales ${ }^{43}$ ?

\section{Le local contre la justice centrale}

13 En forçant un peu le trait, on peut prétendre que, pendant un peu plus d'un siècle, de l'an VIII à la veille du premier conflit mondial, malgré le quadrillage institutionnel et le maillage juridictionnel hérité du « Grand Consulat »" et de l'Empire, le local a conservé, face à la justice étatisée, davantage qu'une autonomie relative. Les sociétés communautaires ont en effet maintenu leurs propres modes de règlement des conflits et de régulation des différends. Dans les consciences collectives, le local l'emporte donc, pendant très longtemps, sur le national. La majorité des justiciables se perçoit comme faisant partie d'un espace vécu : écart, hameau, bourg... Les mouvements de population, c'est-à-dire les migrations définitives qui échappent à l'encadrement des collectivités villageoises ${ }^{45}$, et la guerre de $1870^{46}$ ont déplacé le sentiment d'appartenance en élargissant l'horizon. Le sentiment identitaire étendu à l'ensemble du territoire national l'emporte alors sur les allégeances au local. Les processus de politisation et les apprentissages du politique qui permettent à un électeur d'associer à la fois la vie municipale et l'existence de la communauté nationale y ont bien sûr contribué ${ }^{47}$. Mais il ne s'agit que d'une tendance générale et tardive. La fin des terroirs ${ }^{48}$ est observable entre 1870 et 1914 tandis que certains criminologues scrutent vers 1880 les "conservatoires de l'archaïsme", sortes d'enclaves dans lesquelles les populations n'auraient presque pas changé depuis des temps immémoriaux et échapperaient à l'État central et à sa justice, à l'instar de la Corse étudiée par Albert Bournet ${ }^{49}$.

14 Les anthropologues et les historiens ruralistes, en étudiant les communautés villageoises, qui se définissent à l'aune de la proximité et du point d'honneur, ont plus récemment découvert, à partir des archives judiciaires ${ }^{50}$, la justice concrète, telle qu'elle s'exerçait véritablement. Bigarrée, cette dernière se décline plutôt au pluriel qu'au singulier. Elle mêle en effet usage de la violence, maintien des équilibres, tentatives d'apaisement et bien d'autres traits encore. Schématiquement trois modes de régulation des conflits, dont l'initiative échappe à l'État, s'imbriquent et constituent la justice communautaire: la vengeance, l'arrangement et le recours à la justice officielle qui est une manière de la détourner ${ }^{51}$.

En effet, les sociétés villageoises, et parfois les collectivités de quartier, investissent et instrumentalisent la justice étatisée. Les «comptes moraux » envoyés au XIX ${ }^{\mathrm{e}}$ siècle à la Chancellerie à l'occasion de chaque session de cour d'assises sont une occasion de suivre au quotidien le fonctionnement de la justice. Les rapports des procureurs généraux constituent une autre source qui atteste de la diversité régionale et permet de scruter l'activité journalière de la surveillance. Pour rester dans le domaine du jury, certains « acquittements scandaleux $»^{52}$ démontrent, s'il en était besoin, qu'il n'est pas toujours possible de se soustraire aux pressions locales.

La culpabilité se module selon l'appréciation des jurés mais aussi selon le rang social. D'aucuns affirment même que la justice est au service des "gros ", qu'elle condamne très fortement les auteurs d'attentats contre la propriété et qu'elle protège les familles des notables. Les réseaux clientélaires tissés offrent une protection efficace contre l'intrusion de l'autorité judiciaire. Yves Pourcher a montré que les notables de la Lozère, du moins certains d'entre eux, pouvaient impunément tirer un coup de fusil sur 
un paysan qui refusait que son champ non moissonné puisse devenir le théâtre d'une chasse, donner des coups de crosse à la sœur de l'un d'eux, et tuer un cousin qui venait au secours de ses parents et être acquittés ${ }^{53}$. Ailleurs, dans les campagnes poitevines, un cultivateur de 26 ans échappe aux rets de la justice grâce à son statut. Qualifié de "lovelace de village ", il avait commis en toute impunité des violences sexuelles contre « sept femmes et sept jeunes filles » car il appartenait à une famille de fermiers aisés qui au pouvoir économique et social, avait ajouté le pouvoir municipal. Son cousin était maire de la commune, son oncle adjoint. Il a pu ainsi écarter dix jurés et être acquitté ${ }^{54}$. Ainsi donc, par toutes sortes de moyens, les délibérations du jury ne peuvent se déprendre des passions communautaires.

Mais d'autres inculpés n'attendent pas que l'affaire soit renvoyée devant une juridiction de jugement, c'est dès les premiers pas de l'instruction que leur action se fait sentir. Jusqu'à la fin du Second Empire, dans les campagnes proches de la capitale, plusieurs magistrats instructeurs se plaignent de l'aphasie des témoins. Telle affaire demandera dix ans d'enquête: "la peur parait avoir longtemps enchainée la langue des témoins les plus essentiels ", telle autre exigera du juge une rare patience pour décrypter quelques monosyllabes d'un berger, unique témoin d'un crime et littéralement terrifiés5. Aussi ce ne sont pas seulement les acquittements scandaleux et les indulgences coupables qui sont vilipendés par la justice centrale, ce sont aussi les résultats "de sollicitations et de l'intrigue $»^{56}$. Toutefois quelques cas, colportés avec empressement, montrent que les jurés ne sont pas à l'abri de représailles. Des proches du condamné usent de menaces, écrivent des lettres anonymes dans lesquelles ils se réjouissent de venger leur parent et se promettent de transformer en brasier les biens des membres du jury considérés comme les responsables de la condamnation. Ailleurs encore, un menuisier condamné pour assassinat avait promis de se venger s'il parvenait à s'échapper. C'est chose faite en 1862, et tandis que des nouvelles alarmistes se répandent, les jurés qui le connaissaient personnellement sont avertis par la gendarmerie ${ }^{57}$. Ces quelques exemples suffisent à montrer que la sérénité a déserté les prétoires et que le modèle de justice étatisée et centralisée connaît de multiples adaptations. Dans une population largement rurale - en 1861 on dénombre un peu moins de 27 millions de ruraux pour un peu moins de onze millions d'urbains ${ }^{58}$ - les relations personnelles, constituées en groupe de pression, pèsent d'un poids très important. Le recours à la justice officielle n'est pas guidé par le souci d'équité, on ne demande pas réparation d'un préjudice subi, mais on désire prendre un avantage décisif sur son adversaire. C'est une manière de faire perdre la face à l'autre ou du moins de lui causer le plus de désagréments possibles. Si de semblables pratiques peuvent être observées en Gévaudan, dans le Poitou, ou dans les Pyrénées, on les retrouve aussi en Île-de-France. Un commentaire, aux allures de proverbe, illustre une telle situation : « il a hérité en même temps que des biens des procès ».

La justice est également utilisée pour orienter les magistrats instructeurs dans des directions fort éloignées de la vérité. Les présidents de cours d'assises font part, pendant le XIX ${ }^{e}$ siècle, de leurs doléances, parmi elles, la question des faux témoignages semble récurrente. À les croire, le phénomène est général et nombre de témoins se mettent au service de l'accusé et n'hésitent pas à inventer de véritables fables. Une poignée de magistrats se muent en ethnologues, observent leur ressort et détectent des zones où les faux témoins, condamnés en correctionnel, se rencontrent en plus grand 
nombre. Dans le département de la Vienne, la région de Châtellerault acquiert ainsi une renommée durable, que la presse régionale de la fin du XIX ${ }^{\mathrm{e}}$ siècle confortera.

L'instrumentalisation de la justice étatisée se fait aussi par le haut de la pyramide sociale. Les hobereaux s'adressent à elle à l'occasion de contentieux mineurs. Dans les régions à châteaux du Limousin, là où les demeures de la distinction persistent et s'adaptent, faire appel à la justice fait partie des stratégies de domination des châtelains. Dans 82 \% des affaires du ressort du tribunal de simple police ou de la justice de paix, ce sont ces derniers qui demandent la médiation du juge pour « des questions de créances, de bornage, de droits d'usage ou encore des dégâts commis sur les propriétés ${ }^{59}$. La justice étatisée est employée à maintenir l'ordre au village; le rôle qui lui est dévolu consiste à s'assurer que rien, dans le domaine des relations politiques et sociales, ne puisse véritablement se transformer.

Reste que le recours à la justice, qui parfois s'apparente à une "vengeance officielle ", quelles qu'en soient les logiques, ne peut rester sans incidence. Détournée à des fins privés, utilisée pour assouvir une rancune personnelle, elle est aussi une des manifestations de l'acculturation judiciaire. Michel Alliot avait jadis attiré l'attention sur la vision qu'une "société a du monde et d'elle-même explique, plus particulièrement, les comportements juridiques individuels et (...) les limites de la juridicité $\aleph^{60}$. Lorsque les communautés se fragmentent, que les liens se distendent, les autres modes de régulation se désagrègent. Il n'en reste pas moins qu'une grande défiance perdure. La législation et la réglementation apparaissent pour le "peuple des campagnes » comme venant du monde des villes. La justice, extérieure et lointaine, est urbaine. Elle n'a pas été faite pour nous, pense une majorité de ruraux. Et c'est en 1931 seulement que la population des villes dépassera la population urbaine. Pour s'imposer, la justice étatisée doit être non seulement présente, mais aussi conquérir l'imaginaire des campagnes. Au cours de ces années de l'entre-deux-guerres, dans les prières du soir, on demande à Dieu, dans nombre de communautés villageoises, qu'il les protège de la Justice.

Cette dernière pourtant, s'est montrée à plusieurs reprises réservée, voire timorée. Les représentations d'une justice toute puissante et omniprésente se sont brusquement dérobées pendant le premier XIX ${ }^{\mathrm{e}}$ siècle et même au-delà. Eugen Weber évoque l'âge d'or des bagarres entre villages et signale que la Statistique du département du Lot parle de "guerres qui éclatent entre les différentes communes et donnent lieu à de véritables combats $»^{61}$. En effet, les rixes villageoises, provoquées par le groupe des jeunes, faisaient la démonstration d'une violence inouïe à tel point que chaque année on relevait des blessés et des morts. La justice laissait faire et bien souvent renonçait à toute intervention. Depuis, François Ploux a rouvert le dossier et confirmé que le Quercy avait connu des violences extrêmes qui s'apparentent davantage à des guerres qu'à des rixes $^{62}$. Ailleurs encore, en Bretagne par exemple, en 1866, 300 jeunes s'affrontent ${ }^{63}$, mais après la chute du Second Empire, elles sont plus rares. Toutefois, pendant plus d'un demi-siècle, les pouvoirs publics apparaissent bien souvent impuissants et la justice ne parvient que très difficilement à endiguer de tels éclats de violence, comme semble l'attester le faible nombre des condamnations.

La vengeance également peut être lue comme une manière de nier l'importance du pouvoir d'État. La vengeance, pour les anthropologues, s'inscrit dans des sociétés caractérisées par l'absence de système judiciaire unifié et par l'absence ou la faiblesse d'un État national ${ }^{64}$. Outre les vendetta ${ }^{65}$ qui illustrent le retrait ou l'impuissance d'un État central, la multitude des vengeances privées sont en France autant de coups portés 
à l'institution judiciaire étatisée. Cependant la satisfaction vindicative peut conduire au désastre celui qui, se raillant des lois et des règles, n'hésite pas parfois à assassiner un proche, à incendier une maison ou à commettre d'abominables forfaits. La volonté haineuse de prendre une revanche définitive est alors le principal ressort des conduites. Plutôt que d'en arriver à de telles extrémités, qui mettent en péril l'existence de la communauté, car toute action vindicative appelle réciprocité et rétorsion, on préfère le plus souvent user d'accommodements, parfois englobés dans l'expression générique d'infrajudiciaire ${ }^{66}$.

Accord généralement financier et oral entre deux parties, l'arrangement est une manière de mettre un terme provisoire ou définitif à un conflit qui aurait pu conduire l'un des protagonistes en cour d'assises si les autorités avaient été alertées. L'habitant des campagnes se présente sous les traits d'un « être-en-comptes ». Chacun s'ingénie à tenir l'inventaire des animosités, tandis que tous observent avec une jubilation feutrée leur évolution. Les accommodements peuvent concerner tous les domaines de l'existence, des emprunts et des dettes à la possession d'une bande de terre séparant deux propriétés. Dans l'Orne, à la lisière de la forêt, les plaisirs de l'arrangement structurent les relations et renforcent le lien social ${ }^{67}$. Dans des sociétés où tout le monde se connaît, la renommée suffit souvent à désigner le coupable. Le plus souvent, les arrangements respectent les hiérarchies sociales. Il semble très rare que des accords soient passés entre un tâcheron et un fermier. En revanche, dans quelques affaires de viols, le maître appartenant à l'univers de la "bourgeoisie rurale " peut offrir une compensation pour s'assurer du silence de la victime. La négociation se noue avec la famille qui peut exiger une somme d'argent plus importante afin de constituer une dot à leur enfant, qui dans ses tractations n'a pas été consultée. Dans d'autres circonstances, en 1866, il arrive que toute une communauté, après s'être arrangée, tente de soustraire le coupable à la justice officielle et organise même sa fuite, de la Lozère à la Drôme ${ }^{68}$. Pour sceller un accord, on ne s'adresse pas comme en Languedoc sous l'Ancien Régime à un "seigneur $»^{69}$, mais à un arbitre plus proche : oncle, maire, patriarche du village...

Du Hurepoix aux communautés villageoises côte-d'oriennes, s'exerce bien une «justice informelle communautaire $»^{70}$ qui n'est pas l'apanage d'espaces enclavés ${ }^{71}$. Progressivement pourtant, mais à un rythme lent, la recherche d'une solution négociée perd de sa vitalité. Il n'en reste pas moins que la justice étatisée sera tenue à l'écart de semblables tractations. Paradoxalement, cette dernière va reprendre à son compte le principe de l'arrangement pour réduire le contentieux le plus massif des régions boisées : les infractions forestières des domaines de l'État ou des bois assimilés. De 1825 à 1840 , le nombre de procès-verbaux dressés à l'occasion des délits des eaux-et-forêt est multiplié par 1000. L'explosion devance le code forestier de 1827 et se poursuit sans discontinuer. Vers 1848, le mouvement interrompu à la hausse faiblit et se rétracte. Dans la forêt de Rambouillet, 422 jugements forestiers sont enregistrés pour la seule année 1840, il n'y en aura plus que 32 en 1868. La loi du 18 juin 1859 a amplifié le phénomène. En effet, elle autorise l'administration des forêts «à transiger avant le jugement définitif sur la poursuite des délits et contravention en matière forestière ». D'où une double décrue : le nombre de procès- verbaux dressés annuellement passe, au niveau national, de 46348 en 1871 à 27950 en 1875 et le nombre des transactions avant jugement passe de 22552 à 26 90. De la sorte, les jugements se sont presque tous volatilisés. La transaction s'intègre aux pratiques rurales et s'identifie aux modes «traditionnels" de régulation des conflits. Au début de la Troisième République, 
l'aisance relative des communautés qui ne sont plus autant que par le passé gorgées de bras, l'invention d'une nouvelle forme de répression, qui semble être un décalque des pratiques rurales, parviennent pratiquement à faire disparaitre du paysage pénal une catégorie d'infractions longtemps dominante. L'administration forestière elle-même introduit une faille dans l'économie pénale en acceptant de négocier avec les contrevenants et les délinquants. Pour nombre de contemporains le dépérissement de ce type d'infractions vient d'une entorse inconcevable à l'égard des principes de la justice étatisée ${ }^{72}$ qui, sur d'autres terrains, et selon une scansion décalée, connaît de multiples « assauts».

\section{Les justiciables contre le modèle judiciaire?}

La codification, les décisions de justice, les attendus de jugement, les magistrats, voire l'État lui-même et le pouvoir judiciaire sont l'objet de fortes contestations qui prennent des formes très diverses. L'incendie du Palais de Justice de Paris en 1871 qui vit les archives et la bibliothèque des avocats, la Cour de cassation, les salles d'assises, les chambres correctionnelles disparaître dans les flammes en est l'illustration la plus emblématique $^{73}$. Les moments révolutionnaires, il est vrai, sont des occasions où les animosités à l'égard de la justice s'expriment sans ménagement, notamment depuis le Premier Empire par l'entremise des placards séditieux. Ce sont des affiches anonymes nuitamment apposées, de préférence sur les édifices publics ou dans les rues passantes. Les autorités judiciaires sont les premières alertées. Des procureurs généraux écrivent qu'il y a là, dans le domaine de la surveillance et du maintien de l'ordre public, «des nécessités politiques et judiciaires». En avril 1849 par exemple, tandis que la Seconde République connaît de nouveaux soubresauts, un placard est trouvé à Vervins ${ }^{74}$. Il contient, comme l'indique l'avocat général de la cour d'appel d'Amiens, la «liste des aristocrates qui seront pendus au poteau auquel est attaché un réverbère sur la place du palais de justice». Plusieurs membres de la société judiciaire figurent en bonne place. Plus tard, une fois l'Empire proclamé, le même lieu symbolique est choisi : sur une des bornes du palais de justice un placard séditieux dénonce le régime à l'occasion de la venue dans la ville de Napoléon III $^{75}$. Les placards font référence souvent à des périodes où la vacance des pouvoirs fut manifeste, de la sorte leur seule existence apparaît comme un camouflet pour la justice. Il incarne la "sédition en marche». En soi l'expression placardière peut sembler dérisoire et pourtant elle montre que dans un secteur délimité, la surveillance de l'espace public est dévolue aux parquetiers et que plusieurs gardes des Sceaux leur accordent une grande importance. Mais il s'agit aussi d'une forme de contestation, parmi d'autres, de l'État et de la justice. Les Archives nationales ont conservé un très grand nombre de textes -souvent le support original a été détruit au moment de son interception, et seul le contenu du message a été recopié, ainsi que la correspondance des procureurs généraux avec l'administration centrale du ministère de la Justice. Mais pour saisir leur impact, il faut se déprendre d'une histoire trop linéaire qui analyse les formes de la contestation du passé à l'aune des manifestations les plus contemporaines et qui dès lors, les ignore avec commisération, en les frappant du sceau de l'archaïsme ou du dérisoire. C'est encore l'archaïsme qui est évoqué pour qualifier les conduites de refus. Nombre de sociétés villageoises refusent conjointement l'État, la loi, les gendarmes, les percepteurs et les magistrats. Dans l'Ariège, la guerre des Demoiselles, nom donné aux insurgés affublés de bonnet et d'une longue chemise, en incarne assurément l'archétype ${ }^{76}$. Forme carnavalesque de révolte 
populaire, elle s'étend entre 1829 et 1872 . Si elle possède de multiples facettes, elle est assurément d'abord une révolte contre la codification. Le rejet du code forestier se traduit notamment par l'assassinat de plusieurs agents de l'État. Des gardes forestiers, plus d'une dizaine, sont tués, victimes d'affrontements individuels ou de guet-apens isolés. Leur mise à mort traduit une haine terrible qui s'exprime par d'horribles traitements. Des corps mutilés de brigadiers sont ainsi découverts dans la forêt ou retirés d'une rivière, l'un d'eux a même eu la langue arrachée avant d'être décapité ${ }^{77}$. Jean-François Soulet, reprenant et complétant l'enchaînement des événements, a proposé une interprétation plus réflexive de la guerre des Demoiselles en s'attachant aux logiques d'une "société en dissidence». La "collaboration limitée » des " fonctionnaires » judiciaires qui renâclent représente l'aspect le plus saillant. Cette fois ce ne sont pas les jurés qui sont dénoncés, mais les magistrats, qui plus est, pendant plusieurs années, par le préfet des Hautes-Pyrénées. Lors de la première phase du conflit, la Cour prévôtale est soupçonnée de « complaisance »; les juges des tribunaux de première instance sont vertement tancés pour diminuer systématiquement le montant des amendes et les réduire à une somme dérisoire; bref les juges ne semblent pas avoir d'autres choix qu'opter pour l'incompétence ou la complicité ${ }^{78}$.

Le refus de la codification, c'est d'une certaine façon un combat contre tous ceux qui entendent réglementer l'accès à la forêt. D'autre part, la législation, en retenant la responsabilité de la communauté, contribue à maintenir les édiles locaux à distance du personnel de l'État. Il ne leur est pas possible de partager la même vision du droit englobant et de l'organisation de la société. Aussi peut-on affirmer que comme «les peines collectives demeurent, et <qu'>elles retombent sur les élites municipales, la rancœur de ces dernières ne s'éteindra pas de sitôt $~^{79}$. Les droits d'usage et les biens communaux sont l'objet d'une lente codification qui témoigne d'une volonté étatique, indépendamment des régimes. Si les biens communaux ${ }^{80}$ parlent davantage à l'imaginaire - pour le seul département de Seine-et-Oise ils ne dépassent pas au début du XIX ${ }^{e}$ siècle $0,3 \%$ des superficies occupées - les premiers, encore après la Seconde Guerre mondiale, sont au centre de multiples préoccupations conflictuelles. Dans les circonscriptions administratives, une législation régionale et parfois locale voit le jour afin de compléter les dispositions nationales ou plus pragmatiquement pour combler le vide juridique. Les bornes chronologiques de l'offensive du législateur sont aisément repérables. En amont, les lois du 28 septembre 1791 et 3 Brumaire an IV; en aval, la législation de la fin des années 1880. Le passage d'une règle usuelle à la codification des usages ne s'effectue qu'après modification des pratiques collectives. Aussi les lois du 9 juillet 1889 et du 22 juin 1890 sonnent-elles le glas, par exemple, de la vaine pâture. L'établissement d'une règle écrite ne s'est pas faite sans résistance et sans controverse et les opinions publiques villageoises, parfois unanimes, parfois divisées, ont voué aux gémonies le législateur royal comme le législateur républicain. Il n'en reste pas moins que l'entreprise de codification s'attache dans les campagnes entre 1880 et 1900 aux rapports sociaux. Ce sont les relations contractuelles tacites qui sont métamorphosées en règles juridiques. Que l'on songe aux difficultés d'établissement du code rural ${ }^{81}$ qui pour les juristes est resté inachevé. Toutefois, il n'en reste pas moins que «l'usage local » dans sa dimension agricole définie par la loi du 3 janvier 1924, reste particulièrement vivace. La codification audoise n'intervient qu'au cours des années 1932-1938 ${ }^{82}$.

Les autres codes sont aussi l'objet de vives critiques. Pour certains, ils sont le reflet du despotisme et d'une société patriarcale. Sans entrer plus avant dans cette discussion, 
signalons qu'en matière criminelle, la procédure est au cœur des débats. Même si la loi de 1897 associe l'avocat aux diverses phases de l'instruction, les droits de la défense apparaissent encore singulièrement réduits. Quant au code civil, il cristallise un moment du rapport entre les sexes. N'est-il pas le symbole de l'oppression et de la sujétion des femmes. En fixant des normes en fonction des devoirs d'épouse et de mère, il organise, après les avoir désignées comme un groupe social, l'infériorité juridique des femmes ${ }^{83}$. Par exemple la femme française, séparée de corps, ne retrouvera sa capacité juridique qu'en 1894. Les difficultés pour obtenir un semblant de mesure pour pouvoir conserver, sous certaines conditions, le produit de son travail (loi du 13 juillet 1907) l'attestent. Bref, le code civil est un code contre les femmes. Portalis écrivait qu'il fallait leur offrir une "protection perpétuelle», c'est-à-dire les retrancher de la cité et les cantonner à l'espace privés4, à sa manière il est aussi un des instruments de l'antiféminisme. Christine Bard a rappelé que lors du centenaire du code civil, l'Assiette au Beurre, volontiers libertaire, anticolonialiste et aiguisant d'inlassables traits contre les «gros" et la bêtise, croque néanmoins des portraits de femmes, en «horribles mégères $»^{85}$. Les combats sur le terrain juridique témoignent bien de l'incessante contestation du "Code napoléon » et d'une critique féministe naissante du Droit, même si les relais auprès de l'opinion publique connaissent de multiples embûches comme l'illustre la presse populaire qui se montre peu diserte. Dans cette perspective, il n'est pas anodin de rappeler que Léon Richer, se situe sur le terrain de la loi et du droit. C'est avec le vocabulaire et les armes des juristes qu'il décide de se battre. Après avoir lancé en 1869 le journal féministe le Droit des femmes, il mène une action prioritaire en faveur de la recherche en paternité et du droit au divorce, puis il intitule son œuvre la plus ambitieuse Le code des femmes.

Avec l'avènement de la III République, l'étatisation de la justice prend, dans un premier temps, des allures de couperet, ce sont les grandes épurations judiciaires des années 1879-1883. Malgré le caractère parfois «absolu» du nouveau régime ${ }^{86}$, la démocratie et la transparence se renforcent. De la sorte, la justice centralisée et étatisée devient l'objet, lors de dysfonctionnements, de vives critiques, relayées cette fois par une presse en pleine expansion, protégée de la censure et des poursuites judiciaires, qui manie l'effet d'annonce et traite l'information comme une succession de faits divers. Le Petit Journal, Le Petit Parisien, Le Journal, Le Matin atteignent ou dépassent chacun un million d'exemplaires à la veille de la Première Guerre mondiale. Les "scandales » bénéficient dans l'opinion publique d'échos répétés. C'est à la suite de l'affaire des décorations que le président de la République Jules Grévy présente sa démission en 1887. Son gendre organisateur du trafic est, après une première condamnation, acquitté par la cour d'appel de Paris. L'impartialité de la justice est alors raillée, et l'institution judiciaire, comme d'autres grandes institutions, connaît un discrédit qui n'est pas que conjoncturel. Il participe d'un processus de désacralisation qui s'accélère ${ }^{87}$. Dans des registres différents, les affaires Dreyfus et Stavisky contribuent au discrédit de l'État et de la Justice ${ }^{88}$. Elles soulignent que le modèle judiciaire ne fonctionne pas, ou du moins ne répond pas aux exigences de la démocratie. L'affaire Oustric, encadrée par les deux précédentes, avait entraîné la chute du cabinet Tardieu et conduit plusieurs ministres devant la justice ${ }^{89}$, dont le Garde des sceaux Raoul Péret. Lors de l'affaire Dreyfus, dans sa seule dimension judiciaire, après la découverte en 1898 d'un faux dans le dossier d'accusation, la chambre criminelle de la cour de Cassation est dessaisie et ce sont les chambres réunies qui cassent l'arrêt du conseil de guerre ${ }^{90}$. Quant à son retentissement et à sa réception, 
il n'est guère besoin ici d'insister sur leurs effets. L'affaire Stavisky est à la fois célèbre et méconnue. On sait qu'il menait grand train, qu'il recevait des personnalités de premier plan, qu'il monta en 1933 une escroquerie colossale devant lui permettre d'engranger deux cents millions de francs et qu'il n'était pas inconnu de la justice. Des dix-neuf poursuites judiciaires engagées contre lui aucune n'avait pourtant aboutie. Lorsque l'on retrouve son cadavre, l'opinion publique ne croit pas à un suicide. Mais l'affaire n'est pas close pour autant. Le conseiller Prince, qui avait eu notamment en charge le dossier des escroqueries des prestations en nature dues par l'Allemagne en exécution du Traité de Versailles ${ }^{91}$, était considéré comme un véritable spécialiste des questions financières, et c'est à lui que furent dévolus les documents comptables; il accuse son supérieur, le procureur Pressard, beau-frère du président du Conseil, d'être le responsable de l'enfouissement des poursuites contre Stavisky. Peu après, le Conseiller Prince est à son tour retrouvé mort le long d'une voie ferrée, décapité par un train. L'enquête expertale ne laisse guère de doute sur le fait qu'il s'agit d'un assassinat. C'est pourtant la thèse du suicide qui est retenue. L'événement semble appartenir à la chronique des faits anecdotiques, et pourtant il avive les antagonismes politiques et les ambitions des ligues qui contestent ouvertement la légitimité de l'État, il souligne aussi la collusion de l'État, des milieux financiers troubles et de la justice. Le soupçon de la dépendance se mue en certitude pour une partie de l'opinion : la justice, qui après avoir découvert la " question sociale » l'a oubliée, est aux ordres du pouvoir politique et des « 200 familles ». À peu près au même moment, surgit une critique radicale de l'État, dont des représentants de la société judiciaire se montrent particulièrement actifs. Brassant les thèses de Léon Duguit et de l'École de Bordeaux, critiquant le solidarisme de Léon Bourgeois, vilipendant la pratique des décrets-lois née pendant la guerre, réfléchissant à la constitution d'États "dictatoriaux » en Europe... chacun trouve des références théoriques et puise des arguments à la fois dans de savants traités comme dans la situation politique immédiate, en France comme en Europe. Toujours est-il que les quatre premières décennies du XXe siècle connaissent une remise en cause inégalée de l'État et du modèle judiciaire. Quelques voix s'élèvent pour demander si la justice, qui n'est pas un "pouvoir judiciaire » serait un service public? En 1934, des juristes de renom clament que le « Français n'a plus confiance dans la justice de son pays ».

29 "Il faut tenter de donner à l'État sa juste place »" S'il importe en effet de suivre les manières dont l'étatisation de la justice se met en place, d'en saisir les logiques et les dynamiques, il convient aussi de se défier d'une histoire trop linéaire pour laquelle le modèle d'une justice étatisée et centralisée serait déjà clos en 1810. Le discours juridique, il est vrai, insiste volontiers sur les permanences. La magistrature des cours d'appe ${ }^{93}$ a produit d'une rentrée à la suivante des images sur l'institution judiciaire qui résiste au temps et défit les révolutions, les guerres et les régimes. La justice serait le garant du lien social et l'ultime rempart lorsque l'État menace de s'effondrer et que la société risque de disparaître. Pourtant, les communautés, les individus et les justiciables manifestent une demande de justice à laquelle l'État central n'apporte pas toujours de réponses. Au pénal, la justice communautaire semble pendant longtemps préférable; au civil, dans l'entre-deux-guerres, les justiciables se détournent de la justice étatisée qui n'a certainement pas été achevée avec la Révolution et l'Empire. En déplaçant le regard de l'observateur vers les hommes, les pratiques et les activités 
concrètes des juridictions, le processus de centralisation apparait incomplet, heurté, divers et complexe. De même que le mouvement d'étatisation s'accomplit selon des rythmes décalés, le modèle d'une justice centrale ne peut se comprendre que comme un des éléments, le plus important, d'un pluralisme judiciaire qui n'a jamais cessé d'exister. Quant aux scandales que l'on ne qualifie pas encore " d'affaires », à l'impunité dont bénéficient manifestement politiciens et escrocs, à la place subalterne accordée au " pouvoir judiciaire » depuis les années 1880 , au fonctionnement quotidien de la justice parasitée par son coût dispendieux et ses lenteurs - vieilles antiennes réactualisées - ils sont autant d'éléments qui participent au désenchantement et ébranlent le modèle judiciaire. Si la justice ne peut pas déjà être le "gardien des promesses " ${ }^{94}$, elle n'est pas davantage la sentinelle de la démocratie républicaine. De la première République au début de la II ${ }^{e}$ République, les deux grands principes républicains sont ceux de l'élection des juges et d'une justice de proximité. Le premier est abandonné dès les années 1880, le second est remis en cause, pour la première fois depuis l'an VIII, par le gouvernement Poincaré en 1926. Aux yeux des justiciables et des citoyens, il ne semble pas plus exister de modèle républicain de la justice que de modèle d'une prison républicaine ${ }^{95}$.

\section{BIBLIOGRAPHIE}

Aberdam S., Aux origines du Code rural. 1789-1900, un siècle de débat, Paris, INRA, 1984.

Agulhon M., La République au village. Les populations du Var de la Révolution à la Seconde République, Paris, Seuil, 1970.

Alliot M., Les résistances traditionnelles du droit moderne dans les États d'Afrique francophone et à Madagascar, dans Études de droit africain et malgache, Paris, 1965.

Assier-Andrieu L., (dir.), Les usages locaux en France rurale. Genèse et actualité, 1989, Rapport de recherche, Toulouse, ministère de la Justice.

Association française pour l'histoire de la Justice, Les ministres devant la Justice, Arles, Actes Sud, 1997.

Aubusson de Cavarlay B., Obscurité du nombre, lumière des chiffres, Équinoxe, printemps, 21,1999, pp. 33-46.

Audren F., La justice au risque de l'histoire. Histoire de la justice, 1789-1958 : état de la recherche française, Cahiers Jean Jaurès, 1996, n 142, pp. 25-46.

Baby F., La guerre des Demoiselles en Ariège (1829-1872), Saverdun, 1972.

Badinter R., (dir.), Une autre justice. 1789-1799, Paris, Fayard, 1989.

Badinter R., La Prison républicaine, Paris, Fayard, 1992.

Baillaud B., et al., Un demi-siècle de civilisation française (1870-1915), Paris, Hachette, 1916.

Bard C., (dir.), Un siècle d'antiféminisme, Paris, Fayard, 1999. 
Baruch M.-O, Duclert V. (dir.), Serviteurs de l'État. Une histoire politique de l'administration française. 1875-1945, Paris, La Découverte, coll. L'Espace de l'Histoire, 2000.

Berlière J.-M., L'ordre et la sécurité : les nouveaux corps de police mis en place par la III $^{e}$ République, XXe Siècle-Revue d'histoire, 1993, 39, pp. 23-37.

BerlièreJ.-M., Le monde des polices en France, Bruxelles, Complexe, 1996.

Berstein S., Rudelle O., (dir.), Le modèle républicain, Paris, PUF, 1992.

Burguière A.,Revel J., Histoire de la France. L'espace français, Paris, Seuil, 1989.

Bouchardon P., Le magistrat, Paris, Hachette, 1926.

Bournet A., La criminalité en Corse, Archives d'Anthropologie criminelle, 1888-III.

Cassin R., L'inégalité entre l'homme et la femme dans la législation civile, Marseille, Barladier, 1919.

Castan Y., Honnêteté et relations sociales en Languedoc, 1715-1780, Paris, Plon, 1974.

Castan N., Les criminels de Languedoc. Les exigences d'ordre et les voies du ressentiment dans une société pré-révolutionnaire (1750-1790), Toulouse, Association des publications de l'Université de Toulousele-Mirail, 1980.

Chauvaud F., "La vengeance, l'arrangement et l'arbitrage », dans Les passions villageoises au XIX siècle, Paris, Publisud, 1991, pp. 184-217.

Chauvaud F., Le dépérissement des émotions paysannes dans les territoires boisés au XIX ${ }^{e}$ siècle, in Coll., La terre et la cité, mélanges offerts à Philippe Vigier, Paris, 1994, Créaphis.

Chauvaud, F., (avec la coll. de Yvorel, J.-J.), Le juge, le tribun et le comptable, Histoire de l'organisation judiciaire entre les pouvoirs, les savoirs et les discours (1789-1930), Paris, Economica/Anthropos, 1995.

Chauvaud F., Les violences rurales et l'émiettement des objets au XIX ${ }^{e}$ siècle. Lecture de la ruralité, Cahiers d'histoire, 1997, tome XLII, 1, pp. 49-88.

Chauvaud F., (dir.), Le sanglot judiciaire, Grâne, Créaphis, 1999.

Chauvaud F., Les criminels du Poitou au XIXe siècle. Les monstres, les désespérés et les voleurs, Geste éditions, La Crèche, 1999.

ChevalierL., Classes laborieuses et classes dangereuses, Paris, Plon, 1958.

Claval P., Les freins à l'homogénéisation du territoire, in de Planhol X., Géographie historique de la France, Paris, Fayard, 1988, p. 485 et suiv.

Claverie E., Lamaison P., L'impossible mariage. Violence et parenté en Gévaudan. $17^{e}, 18^{e}$ et $19^{e}$ siècles, Paris, Hachette, 1982.

Claverie E., De la difficulté de faire un citoyen : les « acquittements scandaleux du jury dans la France provinciale du début du XIX ${ }^{e}$ siècle », Études rurales, 1984, 95-96, pp. 143-166.

Clout H., Themes in the Historical Geography of France, New York-San Francisco, Academic Press, 1977.

Coll., Influence du modèle judiciaire français en Europe sous la Révolution et l'Empire, Lille, L'Espace Juridique, Actes du colloque de juin 1998, 1999.

Commaille J., Territoires de justice. Une sociologie politique de la carte judiciaire, Paris, PUF, 2000.

Corbin A., L'histoire de la violence dans les campagnes françaises au XIX ${ }^{e}$ siècle. Esquisse d'un bilan, Ethnologie française, XXI, 3, pp. 224-236. 
Corbin A., Le village des cannibales, Paris, Aubier, 1991.

Corvol, A., L'homme aux Bois, Paris, Fayard, 1987.

Delbez L., Du territoire dans ses rapports avec l'État, Revue générale de droit international public, 1932, p. 707 et suiv.

Démier F., Aux origines économiques de la ligne Magiolo, dans La terre et la Cité, mélanges offerts à Philippe Vigier, Paris, CREAPHIS, 1994, pp. 231-249.

Desplat C., La guerre oubliée. Guerres paysannes dans les Pyrénées (XII ${ }^{e}-X I X^{e}$ siècles), Biarritz, J.\&D. éditeurs, 1993.

Donavan J.-M., Infanticide and the juries in France, 1825-1913, Journal of Family History, 1991, 16, 2, pp. 157-176.

Duclert V., Jaurès, la justice et l'affaire Dreyfus, Cahiers Jean Jaurès nº141, 1996, 141, pp. 119-122.

Dupâquier J., (dir.) Histoire de la population française. 3. De 1789 à 1914, Paris, PUF, 1988.

Dupont-Bouchat M.-S., Pierre É., (dir.), Enfance et justice au XIX ${ }^{e}$ siècle, Paris, PUF, Droit et justice, 200.

Emsley $\mathrm{Cl}$., The Nation-State, The Law and the Peasant in Nineteenth-century Europe, in Rousseaux X., Lévy R.,(dir.), Le pénal dans tous ses États, Justice, États et sociétés en Europe (XII ${ }^{e}-X X^{e}$ siècles), Bruxelles, publications des Facultés universitaires Saint-Louis, 1997, pp. 153-178.

Favre J., La justice à Paris pendant le siège de la Commune (1870-1871), Marchal et Godde, Paris, 1919.

Farcy J.-C., Deux siècles d'histoire de la justice en France (1789-1989), Paris, CNRS Éditions, CD-Rom, 1996.

Farcy J.-C., Magistrats en majesté. Les discours de rentrée aux audiences solennelles des cours d'appel (XIX'-XXe siècles), Paris, CNRS Éditions, 1998.

Ferron L., Le rôle du jury et les peines prévues pour les viols et les attentats à la pudeur, La répression pénale des violences sexuelles au XIX ${ }^{e}$ siècle : l'exemple du ressort de la cour d'appel d'Angers, Université d'Angers, thèse dac., janvier 2000.

Fontaine L., Gestion des conflits et contrôle social des travailleurs migrants originaires des montagnes françaises (fin XVII ${ }^{e}$ siècle-milieu du XIX ${ }^{e}$ siècle », Crime, Histoire \& Sociétés, 1997, 1, 1, pp. 71-85.

Fraisse G., Perrot M., (dir.), Histoire des femmes en Occident. 4 Le XIXe siècle, Paris, Plon, 1991.

Garapon A., Le gardien des promesses. Justice et démocratie, Paris, Odile Jacob, 1996.

Garnot B., (dir), L'infrajudiciaire du Moyen-Âge à l'époque contemporaine, Paris, Dijon EUD, 1996.

Gide P., Étude sur la condition privée de la femme, Paris L. Larose et Forcel, 1885.

Grancoing Ph., Les demeures de la distinction. Châteaux et châtelains au XIX ${ }^{e}$ siècle en Haute-Vienne, Limoges, PULIM, 1999.

Guérin de la Grasserie R., De la justice en France et à l'étranger au XX $X^{e}$ siècle. Évolution, comparaison, critique, réforme (Études de droit, de législation comparée et de sociologie), Paris, L. Rec. Sirey, 1914, 3 vol.

Guilard J.-Y., Les pratiques infrajudiciaires des communautés villageoises côte-d'oriennes du XIX ${ }^{e}$ siècle, in Garnot B., (dir.), L’infrajudiciaire, Paris, Dijon, 1996. 
Guionnet C., L'apprentissage de la politique moderne. Les élections municipales sous la monarchie de Juillet, Paris, l'Harmattan 1997.

Jaume L., Les libéraux français et le jury criminel, Tocqueville Review, 1997, 18, 2, pp. 49-70.

Joly H., La France criminelle, Paris, Cerf et fils, 1889.

KalifaD., L'encre et le sang. Récits de crime à la Belle Époque, Paris, Fayard, 1995.

Lascoumes P., Poncela P., Lenoël P., Les grandes phases d'incrimination. Les mouvements de la législation pénale 1815-1940, Paris, ministère de la Justice, 1992.

Lentz T., Le grand Consulat. 1799-1804, Paris, Fayard, 1999.

Levasseur E., La population française, 1891, 2.

Machelon J.-P., La République contre les libertés ? Les restrictions aux libertés publiques de 1879 à 1914, Paris, PFNSP, 1976.

Martinage R., Royer J.-P., Les destinées du jury criminel, Lille, L’Espace juridique, Hellemmes, Ester, 1990.

Maxwell J., Le crime et la société, Paris, Ernest Flammarion, 1924.

Muchembled R., Le temps des supplices. De l'obéissance sous les rois absolus. XV ${ }^{e}-X V I I I^{e}$ siècles, Paris, Armand Colin, 1992.

Ozouf-Marignier M.-V., La formation des départements. La représentation du territoire français à la fin du XVIII siècle, Paris, 1989.

Petit J.-G., Théâtre et discours de la violence : les canards parisiens au XIX ${ }^{e}$ siècle, I.A.H.C.C.J. Newletter, octobre 1982, 6, pp. 27-33.

Petit J.-G., Ces peines obscures. La prison pénale en France (1780-1875), Paris, Fayard 1990.

Petit J.-G., Chauvaud F. (dir.), L'Histoire contemporaine et les usages des archives judiciaires, Paris, Honoré Champion, 1998.

Petit J.-G., La justice et l'histoire contemporaine en France, Historia contemporanea, 1999, XVII.

Ploux F., Rixes intervillageoises en Quercy (1815-1850), dans Violence, brutalité, barbarie.-Ethnologie française, 1991, 3, pp. 269-275.

Pourcher Y., Des assises de grâce ? Le jury de la cour d'assises de la Lozère au XIX ${ }^{e}$ siècle, dans Études rurales, 1984, 95-96, pp.174-177.

Retail L., Principes et cadre juridique de l'expertise judiciaire, Paris, Sirey, 1951.

Robert Ph., Évolution historique et enjeux actuels des frontières entre public et privé dans le domaine de la sécurité, in Coll., Ville et hospitalité, Paris, Maison des sciences de l'homme, 1997, pp. 153-172.

Rosanvallon P., L'État en France de 1789 à nos jours, Paris, Seuil, 1990.

Rouet G., Justice et justiciables aux XIXe et XXe siècles, Paris, Belin, 1999.

Rousseaux X., Lévy, R., (dir.), Le pénal dans tous ses États. Justice, États et Sociétés en Europe (XII ${ }^{e}-X X^{e}$ siècles), Bruxelles, publications des Facultés universitaires Saint-Louis, 1997.

Rousseaux X., Dupont-Bouchat M., Vael C., (eds), Révolutions et justice pénale en Europe. Modèles français et traditions nationales, 1780-1830, Paris, l'Harmattan, 1999.

Royer J.-P., Histoire de la justice en France, Paris, PUF, 1995. 
Rudelle O., La République absolue, Paris, Publications de la Sorbonne, 1986.

Schnapper B., Pour une géographie des mentalités judiciaires. La litigiosité en France au XIX ${ }^{e}$ siècle, Annales E.S.C.,1979, 34, pp. 399-419, repris dans Voies nouvelles en Histoire du droit. La justice, la famille, la répression pénale (XVI ${ }^{e}-X^{e}$ siècles), Paris-Poitiers, PUF, 1991, pp. 395-419.

Schnapper B., Le jury criminel, in Badinter R. (dir.), Une autre justice 1789-1799, Paris Fayard, 1989, pp. 149-170.

Schnapper B., De la charité à la solidarité. L'assistance judiciaire française. 1851-1972, Revue d'histoire du droit, 1984, II, pp. 105-150, repris dans Voies nouvelles en histoire du droit, p. 435 et suiv. Soulet J.-F., Les Pyrénées au XIXe siècle, 2-Une Société en dissidence, 1987, Toulouse, Eché.

Tillier A., L'infanticide devant les cours d'assises en Bretagne (1825-1865), Histoire de la Justice, 1997, 7, pp. 117-143.

Thibon C., Pays de Sault. Les pyrénées audoises au XIX siècle : les villages et l'État, Paris, Éditions du CNRS, 1988.

Tarde G., Philosophie pénale, Lyon, A. Storck, 1891.

Tocqueville A. de, De la démocratie en Amérique, $2^{e}$ partie, chap. VIII, 1835.

Vallaud D., Le crime d'infanticide et l'indulgence des cours d'assises en France au XIX ${ }^{e}$ siècle, Informations sur les sciences sociales, 1982, 21, 3, pp. 475-499.

Verdier R., dir., La vengeance dans les sociétés extra-occidentales, Paris, Cujas, 1980.

Vivier, N., Propriété collective et identité communale. Les biens communaux en France. 1750-1914, Paris, Publications de la Sorbonne, 1998.

Weber E., La fin des terroirs. La modernisation de la France rurale. 1870-1914, Paris, Fayard/Éditions Recherches, 1983.

Wilson S., Feuding, Patronage and the Courts in Nineteenth-Century Corsica, in Rousseaux, X., Lévy R., (dir.), Le pénal dans tous ses États, Justice, États et Sociétés en Europe (XII ${ }^{e}-\mathrm{XX}^{e}$ siècles), Bruxelles, publications des Facultés universitaires Saint-Louis, 1997, pp. 209-218.

\section{NOTES}

2. La présente étude prolonge et complète celle de Jacques-Guy Petit, «La Justice en France, 1789-1939. Une étatisation modèle?».

3. Badinter (1989).

4. L'expression «système des tribunaux » apparaît lors du grand débat qui aboutira à la grande loi de 1790 .

5. Bouchardon (1926, pp. 10-11).

6. Pour en prendre la mesure, $c f$. Farcy (1996), Audren (1996) et Petit (1999).

7. Baillaud et al. (1916); Royer (1995, p. 129).

8. Parmi une bibliographie maintenant assez nourrie, $c f$. Muchembled (1992).

9. Rosanvallon (1990).

10. Berstein, Rudelle (1992).

11. Rousseaux, Dupont-Bouchat, Vael (1999).

12. Coll. (1999).

13. Depuis 1998, se tient à l'EHESS un séminaire sur le thème « Servir l'État en France, XIX ${ }^{\mathrm{e}}-\mathrm{XX}^{\mathrm{e}}$ siècles ", voir l'ouvrage collectif qui en est issu : Baruch, Duclert (2000). 
14. Il est toutefois significatif que la seule étude d'ensemble date de 1977 et qu'elle n'ait toujours pas été traduite en français : Clout (1977). Cf., dans une autre perspective, Burguière, Revel (1989). 15. Cf. notamment Delbez (1932, p. 707).

16. Chauvaud (1995), Commaille (2000).

17. Sur le département, $C f$. Ozouf-Marignier (1989).

18. $C f$. en particulier Joly (1889). Cf. aussi les travaux de Michelle Perrot, Bruno Aubusson de Cavarlay et Philippe Robert.

19. Schnapper (1979).

20. Schnapper (1984).

21. Sur l'interprétation statistique, Cf. Aubusson de Cavarlay (1999). Sur «La statistique judiciaire, son histoire et ses usages scientifiques ", $C f$. le numéro spécial que la revue Déviance et société lui a consacrée, juin 1998, vol. 22, n² 2.

22. Schnapper (1979, p. 410).

23. Claval (1988).

24. Démier (1994, p. 244).

25. Rouet (1999).

26. Idem, pp. 349-350.

27. Donavan (1991); Vallaud (1982).

28. Cf. Chevalier (1958) et Kalifa (1995); $c f$. également la contribution pionnière de Petit (1982).

29. Cf. par exemple Berlière (1993, pp. 23-37).

30. Sur certains de ces aspects, $c f$. les travaux de Philippe Robert (1997, pp. 153-172).

31. Gazette des tribunaux, 6 février 1829.

32. Schnapper (1989); Martinage, Royer (1990).

33. Sur ces aspects, voir Lascoumes, Poncela, Lenoël (1992).

34. Archives nationales, $\mathrm{BB} / 20 / 82-1836$.

35. Maxwell (1924, p. 136).

36. Tocqueville (1835); Jaume (1997).

37. Tarde (1891, p. 443 et suiv.).

38. Sur l'infanticide, Cf. note 26 et Tillier (1997, pp. 117-143).

39. Ferron (2000, p. 476).

40. Cf. Émile Levasseur qui proposera également un taux de criminalité pour 100000 habitants qui divise aussi la France en deux (1891, p. 461).

41. Bernard Schnapper (1988, pp. 9-17) précise que le dernier reproche adressé aux cours d'assises est qu'elles se caractérisaient par «l'incertitude et la diversité de leur politique » et qu' » elles constituaient (...) sûrement une jurisprudence « fantasque ».

42. Guérin de la Grasserie (1914).

43. L. Pelatant, De l'organisation de la police : étude historique, théorique et pratique, 1899, p. 271, cité par Berlière (1996, p. 87).

44. Lentz (1999).

45. Les migrations saisonnières n'échappent pas au contrôle des communautés, $c f$. Fontaine (1997).

46. Sur certains de ses aspects, voir Corbin (1991).

47. Se reporter bien sûr à Agulhon (1970); et plus récemment Guionnet (1997).

48. Weber (1983).

49. Bournet (1888-III, p. 5). Cf. aussi Wilson (1997, pp. 209-218).

50. Chauvaud, Petit (1998).

51. Chauvaud (1995, pp. 184-217).

52. Claverie (1984, pp. 143-166).

53. Pourcher (1984, pp. 174-177).

54. Chauvaud (1999, p. 309 et suiv). 
55. Archives départementales des Yvelines et de l'Ancien département de Seine-et-Oise, U cour d'assises, doss. de proc. 1820 et Archives nationales BB/20-1861.

56. Archives nationales, $\mathrm{BB} / 20 / 82-1835$.

57. Archives départ des Yvelines, URA 416, 1862.

58. Dupâquier (1988, pp. 130-132).

59. Grancoing (1999, p. 362).

60. Alliot (1965).

61. Weber (1983, p. 93).

62. Ploux (1991, pp. 269-275).

63. Archives nationales $\mathrm{BB} / 30$ 386-1867.

64. Verdier (1980).

65. Cf. sur ce thème essentiel le colloque organisé par l'IAHCCJ en 1999 (l'appel à communications a été publié dans Crime, Histoire \& Sociétés, n² 2, vol. 2, 1998, pp. 139-141).

66. Garnot (1996).

67. Corbin (1998, p. 139 et suiv.).

68. Claverie, Lamaison (1982, p. 269).

69. Cf. les travaux d'Yves Castan (1974) et de Nicole Castan (1980).

70. Guilard (1996, pp. 427-436).

71. Pour une mise en perspective européenne, voir Emsley (pp. 153-178). Pour la France, Corbin (1991, pp. 224-236), Chauvaud (1997, n¹, pp. 49-88) et Farcy (pp. 191-207).

72. Chauvaud (1994, pp. 101-114).

73. Favre (1919).

74. Archives nationales, $\mathrm{BB} / 30 / 359-1849$.

75. Archives nationales BB/30/405 - 1863.

76. Baby (1972); Desplat (1993).

77. Soulet (1987, p. 515) et Thibon (1988).

78. Soulet (1988, p. 271 et suiv).

79. Corvol (1987, pp. 206 et 225).

80. Vivier (1998).

81. Cf. Aberdam (1984). Au lendemain de la Seconde Guerre mondiale, le code rural comprend, pour l'essentiel, deux grandes lois : celle du 20 août 1881 et celle du 8 avril 1898 (Dalloz, Nouveau Répertoire, t. I, 1947, p. 538).

82. Assier-Andrieu (1989).

83. Fraisse, Perrot (1991); Cf. aussi un « classique » Cassin (1919).

84. Gide (1885).

85. Bard (1999, p. 25).

86. Rudelle (1986) et Machelon (1976).

87. Chauvaud (1999).

88. Il faudrait également ajouter les campagnes contre les «bagnes pour enfants ", d'autant que « seules les maisons correctionnelles relèvent uniquement de l'État », cf. Dupont-Bouchat, Éric Pierre (2001).

89. Association française pour l'histoire de la Justice (1997).

90. Vincent Duclert est aujourd'hui le meilleur spécialiste de l'affaire Dreyfus, dans sa composante archivistique comme dans sa dimension judiciaire. Parmi une vaste bibliographie, $c f$. en particulier Duclert (1994).

91. Retail (1951, p. 10).

92. Rousseaux, Lévy, (1997, p. 14).

93. Farcy (1998).

94. Garapon (1996).

95. Sur la prison en général, Petit(1990); sur la prison républicaine, Badinter (1992). 


\section{RÉSUMÉS}

Incarnation d'un modèle d'État centralisé et d'une rationalisation de la justice depuis 1789 , le modèle français n'a pourtant jamais fait l'unanimité. Résistance et désobéissance constituent quelques-unes des formes d'opposition à « l'imposition » d'un modèle de justice. Aussi, l'image du «bloc de granit» napoléonien se trouve-t-elle fortement ébranlée. L'étude de la géographie judiciaire montre que «l'unification » des territoires de justice n'est pas totalement réalisée. La France a conservé et inventé des divisions régionales, une grande ligne de fracture et des comportements divers ainsi que l'atteste la diversité des conduites des justiciables. La justice concrète se décline plutôt au pluriel qu'au singulier. Au pénal, la justice est parfois instrumentalisée tandis que perdurent d'autres formes de régulation des conflits, à l'instar de l'accomodement. Quant au modèle judiciaire, il est l'objet de très fortes contestations : conduites de refus, critiques des codes, défiance de plus en plus grande à l'encontre de la justice dans le sillage des « scandales » qui ébranlent la société et l'État.

Although it has been the very embodiment of a centralised state and of a streamlined justice system since 1789, the French model has never been the object of unanimity. Resistance and disobedience have been just two of the forms of «opposition » to the imposition of a model of justice, thereby shaking the image of the Napoleonic "granite block». A look at the judicial geography shows that the "unification" of the judicial territory has never been fully accomplished. France has kept her old regional divisions and invented new ones, a major dividing line as well as different behaviours also remain, as shown by the ways in which subjects of the law conduct themselves. On the ground, the justice system is in fact the justice systems. In criminal law, justice is sometimes instrumentalised, alongside other means of conflict resolution, such as arrangements. The judicial model is itself strongly contested: behaviours indicative of rejection, criticism of the codes of law, growing suspicion of the justice system itself in the wake of the « scandals » that have rocked the French society and State.

\section{AUTEUR}

\section{FRÉDÉRIC CHAUVAUD}

Université de Poitiers, UFR Sciences humaines et Arts, 8, rue Descartes, 86022 Poitiers cedex, frederic.chauvaud@wanadoo.fr

Frédéric Chauvaud, professeur d'histoire contemporaine à l'Université de Poitiers, travaille plus particulièrement sur l'histoire de la justice, de la violence et des conflits aux XIX ${ }^{\mathrm{e}}$ siècle et dans la première moitié $\mathrm{du} \mathrm{XX}^{\mathrm{e}}$ siècle. Parmi ses dernières publications : (dir) Le sanglot judiciaire, la désacralisation de la justice (VIII ${ }^{-}-X X^{\mathrm{e}}$ siècles), Créaphis, Grâne, 1999; Les criminels du Poitou au XIX ${ }^{\mathrm{e}}$ siècle. Les Monstres, les désespérés et les voleurs, La Crèche, Geste Éditions, 1999 et Les experts du crime. La médecine légale en France au XIX' ${ }^{\mathrm{e}}$ siècle, Paris, Aubier, Coll. historique, 2000. 